\title{
Endoscopic Stenting of J Pouch Crohn's Stricture After Proctocolectomy
}

\author{
Ajit Pai, MCh, Jan P. Kaminski, MD, John J. Park, MD, Slawomir J. Marecik, MD, \\ Leela M. Prasad, MD
}

Division of Colon and Rectal Surgery, Advocate Lutheran General Hospital, Park Ridge, IL, USA (Drs. Pai, Park, Marecik, and Prasad); Department of General Surgery, UIC Metropolitan Group Hospitals Residency Program, Chicago, IL, USA

(Dr. Kaminski).

\begin{abstract}
Introduction: Crohn's stricture of the ileal J pouch is an important cause of pouch failure and a difficult condition to treat endoscopically. We seek to document the successful endoscopic treatment of fibrostenotic Crohn's strictures of the afferent limb of the ileal $\mathrm{J}$ pouch in a typical patient.

Case and Technique Description: Endoscopic stenting of the strictured segment was performed with a special covered esophageal wall stent, with the technique being refined over multiple episodes of stenting. Ultimately, a partially covered nitinol double-flared esophageal stent led to the optimal outcome.

Discussion: Successful pouch salvage, good quality of life, and avoidance of ileostomy for the past 7 years were achieved. Endoscopic stenting is an important technique in the armamentarium of the surgeon and gastroenterologist to effectively treat fibrostenotic Crohn's strictures of the ileoanal pouch and can successfully avoid the need for complex abdominal procedures, pouch takedown, and permanent ileostomy.
\end{abstract}

Key Words: J pouch, Proctocolectomy restorative, Crohn's disease, Stricture, Stents.

Citation Pai A, Kaminski JP, Park JJ, Marecik SJ, Prasad LM. Endoscopic stenting of J pouch crohn's stricture after proctocolectomy. CRSLS e2014.00052. DOI 10.4293/CRSLS.2014.00052.

Copyright (c) 2014 SLS This is an open-access article distributed under the terms of the Creative Commons Attribution-Noncommercial-ShareAlike 3.0 Unported license, which permits unrestricted noncommercial use, distribution, and reproduction in any medium, provided the original author and source are credited.

A.P. would like to thank the Foundation for Surgical Fellowships for providing the opportunity to undertake fellowship training in minimally invasive and robotic colorectal surgery at the institution of affiliation.

Address correspondence to: Ajit Pai, MCh, Advocate Lutheran General Hospital, Division of Colon and Rectal Surgery, 1775 Dempster Street, Park Ridge, IL 60068, E-mail: dr.ajitpai@gmail.com

\section{INTRODUCTION}

Restorative proctocolectomy with ileal pouch anal anastomosis is now a standard operation for ulcerative colitis, with excellent results. It remains, however, a complex procedure with defined complications that can result in pouch failure. Crohn's disease (CD) of the ileal pouch reservoir is a well-documented albeit uncommon entity that can be an indication for pouch excision or morbid abdominal corrective operations. Endoscopic methods to ameliorate these strictures are not well documented, and we report what we believe is the first documented case of successful management of an afferent limb Crohn's stricture with a partially covered self-expanding metal stent, with long-term follow-up.

\section{TECHNIQUE AND CASE DESCRIPTION}

A 55-year-old man with a presumed history of ulcerative colitis underwent restorative proctocolectomy with ileoanal pouch anastomosis in 1993. He developed small bowel obstruction in 2004, and small bowel resection was performed for stricture disease. Pathology from the specimen diagnosed $\mathrm{CD}$.

In 2005 , the patient returned with symptoms of abdominal distension and decreased oral intake. Abdominal computed tomography demonstrated small bowel obstruction. Flexible sigmoidoscopy at this time revealed a proximal afferent limb stricture within the pouch (Figure 1 and 2). The patient underwent exploration and stricturoplasty. The stricturoplasty resulted in only minimal relief of symp- 
toms, as the pouch restrictured within 6 months. The only option at this point was pouch excision. However, the patient was very reluctant to undergo the advised permanent end ileostomy. For this reason, placement of a flexible wall stent across the strictured segment was considered for dilation and management of the stricture.

Initially, an 18-mm (inner diameter) by 12-cm Polyflex Wallstent (Boston Scientific Corporation, Natick, MA) was used after balloon dilation with good results. When the second stent was placed, endoclips were placed at the distal end to prevent proximal migration (Figure 3). The patient required periodic stent adjustments and replacements every 5 to 6 months. In early 2013, he developed another afferent limb stricture $10 \mathrm{~cm}$ proximal to the known one; repeated stent placements with the Wallstent were not successful, because the stent tended to migrate despite reversal of the flared end and fixation with clips and sutures, and the proximal bowel intussuscepted into the stent (Figures 4-7). An Evolution (Cook Medical, Bloomington, IN) partially covered nitinol double-flared stent with a 20-mm diameter at the covered portion, with $25-\mathrm{mm}$ flared ends, $12.5 \mathrm{~cm}$ in length, was used, and it has neither migrated nor occluded since placement (Figure 8). This has provided the patient with long-lasting relief from his symptomatic stricture. The use of endoscopic stenting has allowed the patient to retain his pouch and avoid permanent ileostomy for the past 8 years.

\section{DISCUSSION}

Restorative proctocolectomy with ileal pouch anal anastomosis is now a standard operation for ulcerative colitis, with excellent results. Pouch failure, requiring permanent diversion or pouch excision, occurs in about $8.5 \%$ of patients when followed for $>5$ years. ${ }^{1}$ The most frequent causes of pouch failure in a large series from the Mayo Clinic were pelvic sepsis, gross fecal incontinence, unacceptably high stool frequency, and change in diagnosis to $\mathrm{CD} .{ }^{2} \mathrm{CD}$ of the ileal $\mathrm{J}$ pouch is relatively uncommon, with a reported frequency between $2.7 \%$ and $13 \% .^{3}$ There are 3 situations in which $\mathrm{CD}$ may occur in the pouch: (1) restorative proctocolectomy with ileoanal pouch anastomosis for documented $\mathrm{CD}$ of the colon, with no evidence of small bowel or anal disease (intentional); (2) proctocolectomy for presumed preoperative diagnosis of ulcerative colitis or indeterminate colitis, in which the specimen reveals CD (incidental); and (3) de novo or true $\mathrm{CD}$ of the pouch developing years after the procedure (delayed diagnosis). The former 2 situations are classified as early-onset CD, as distinct from the latter, which represents late-onset CD of the pouch. 4,5
Pouch retention rates are worse in patients with delayed diagnosis compared with those with intentional or incidental pouch formation, such that 10 -year retention was $71 \%$ overall, $85 \%$ with intentional pouch formation, $87 \%$ with incidental pouch formation, and 53\% with delayed diagnosis. ${ }^{5}$

$\mathrm{CD}$ of the pouch may present as fibrostenotic, fistulizing, and inflammatory variants. ${ }^{3}$ The diagnosis of this condition is difficult because it must be distinguished from other more common complications, including inflammatory pathologies such as pouchitis and "cuffitis." Ischemic stricture is an important differential diagnosis when considering fibrostenotic disease. A combination of history, clinical examination and examination under anesthesia, endoscopy, computed tomography, and histologic specimens may be necessary to clinch the diagnosis.

Clinically, CD of the pouch may be diagnosed in patients with ulceration of the small bowel including the afferent limb without diffuse pouchitis that persists after at least 4 weeks of antibiotics. Stricture formation in the small bowel (including the afferent limb), the pouch, or the pouch inlet combined with ulceration or inflammation in patients not taking nonsteroidal anti-inflammatory drugs is also regarded as $\mathrm{CD}$, as is fistulation after ileostomy closure. $^{5}$

In a large review, Melton et $\mathrm{al}^{6}$ found that those with delayed diagnoses of $\mathrm{CD}$ of the pouch were likely to be associated with a 3-stage procedure, preoperative colorectal stricture, perianal fistula, anal fissure, mouth ulceration, and younger age. The greater the number of these in any patient, the greater the risk for a subsequent delayed diagnosis of $\mathrm{CD}$ of the pouch.

The treatment of fibrostenotic CD is not well documented, because of the rarity of this presentation. Various options, including pouch stricturoplasty, pouch excision, and redo pouch and permanent ileostomy, have all been used. Transabdominal approaches are technically demanding and prone to complications, especially in the setting of active CD. In the largest series of endoscopic therapy of ileal pouch strictures (CD associated and not CD associated), the 5-, 10-, and 25-year pouch retention rates after endoscopic therapy were $97 \%, 90.6 \%$, and $85.9 \%$, respectively. ${ }^{7}$ The authors concluded that recurrence of strictures after resection and anastomosis and stricturoplasty was high, particularly in those with underlying $\mathrm{CD}$, and that endoscopic therapy with balloon dilation and needleknife stricturoplasty, although safe and efficacious, has poorer results when the strictures are secondary to CD. They also noted that not all pouch strictures lead to pouch 


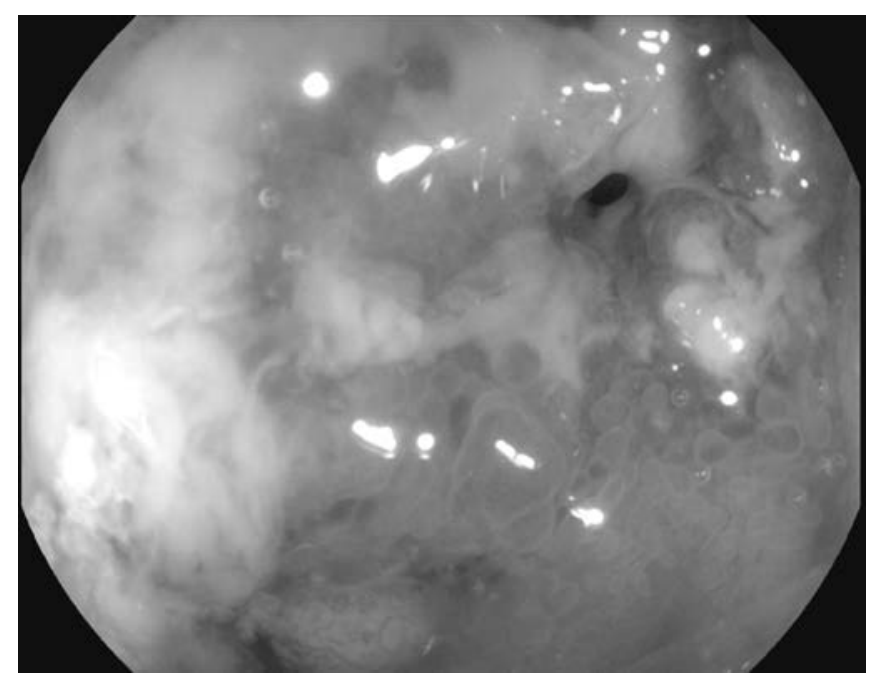

Figure 1. Extensive afferent limb ulceration.

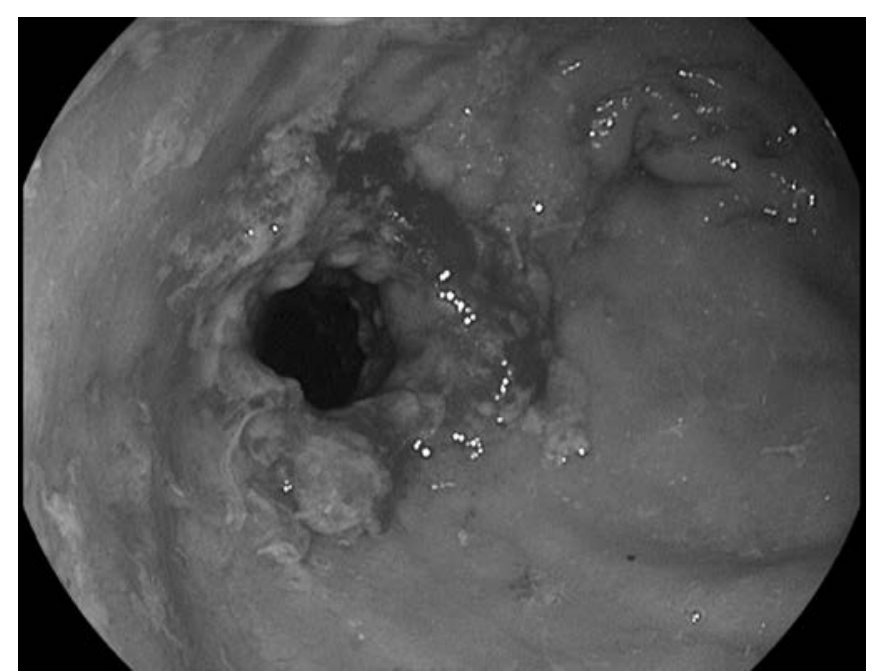

Figure 2. Active ulcerated afferent limb stricture.

failure, although persistent strictures, particularly those related to $\mathrm{CD}$ of the pouch, are among the most common causes of pouch failure. In a large series from the Mayo Clinic, Galanduk et $\mathrm{al}^{8}$ reported a $52 \%$ recurrence rate after simple dilatation of pouch strictures with a Hegar's dilator under anesthesia. A meta-analysis of 13 studies of a total of 347 patients with CD showed that endoscopic dilatation was applied mainly to postsurgical strictures, being technically successful in $86 \%$ of the cases. Long-term clinical efficacy, however, was achieved in only $58 \%$ of the patients, with a mean follow-up period of 33 months. 9

Self expanding metal stents are an attractive nonsurgical modality that have proven efficacy in the treatment of malignant bowel obstruction, either definitive for pallia-

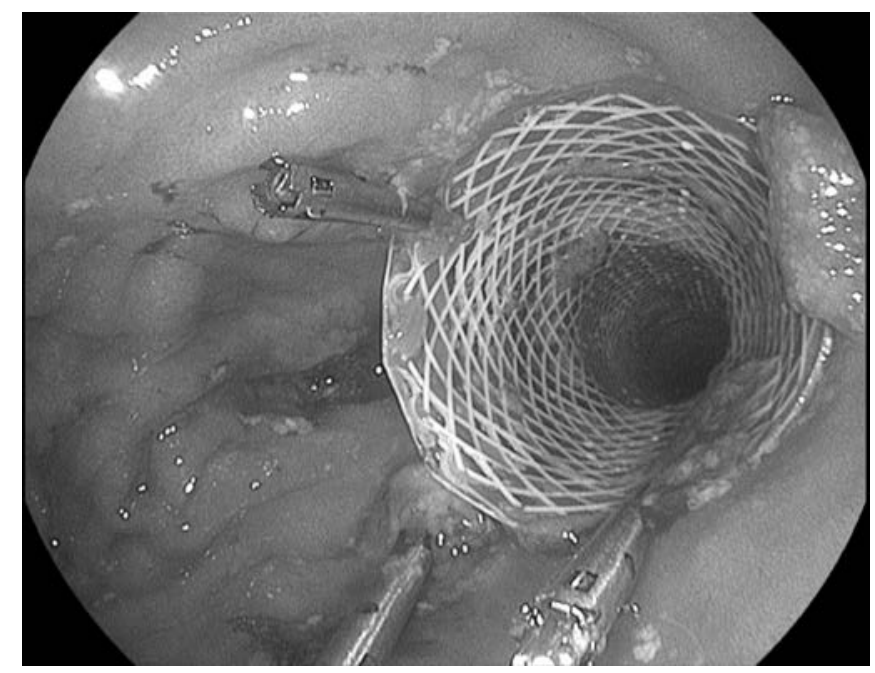

Figure 3. Polyflex single-flare stent with clip at lower end.

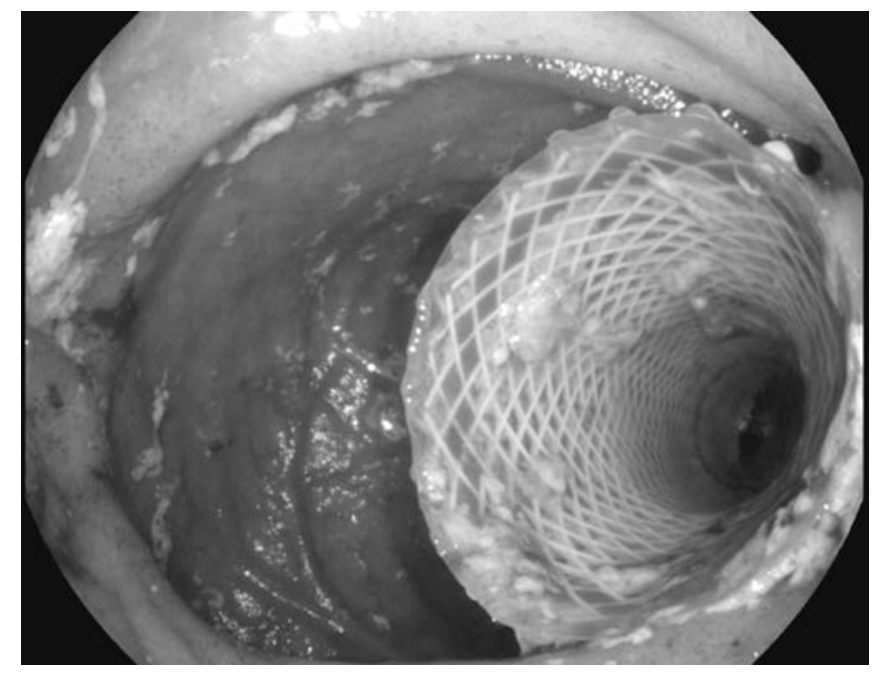

Figure 4. Proximal migration of single-flare stent.

tion or as a bridge to surgery. ${ }^{10}$ The role of these stents in benign obstructions, especially in CD, is controversial, a situation complicated by a paucity of studies, small studies, and limited long-term follow-up. In a series of 5 stented CD anastomotic ileocolonic strictures with the longest follow-up period being 109 months, Levine et al ${ }^{11}$ noted technical success in all patients and complication in only 20\% patients (reobstruction needing surgery). None of the other 4 patients needed further reintervention after stenting. The authors in their literature review of 9 studies with 12 patients and 15 procedures comprising all sites of CD strictures, including terminal ileal, ileocolonic, left colic, and rectosigmoid strictures, also noted that their results were much better than those quoted in other studies, with reintervention needed in $73 \%$ of patients and 


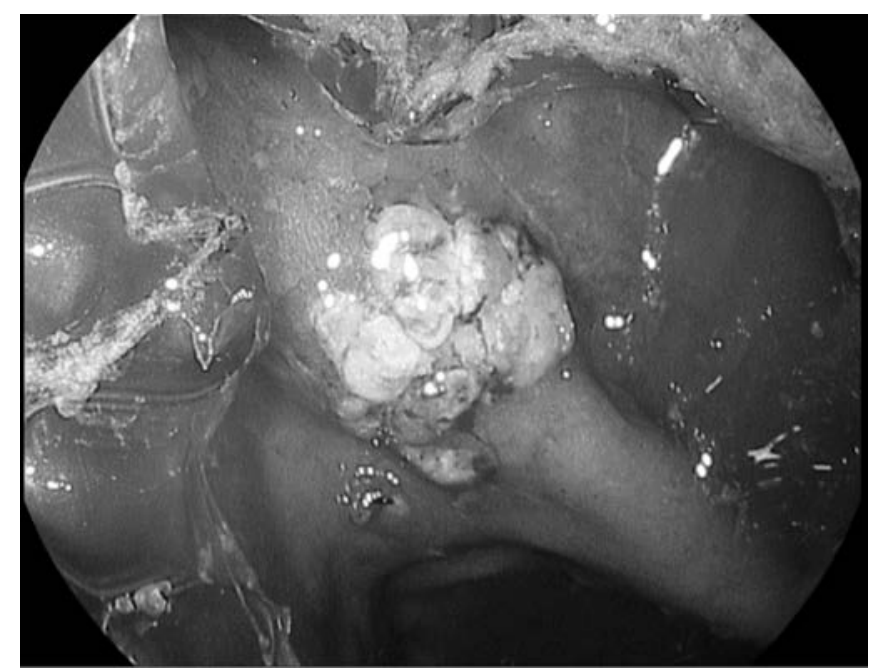

Figure 5. Intussusception of small bowel into the single-flare stent.

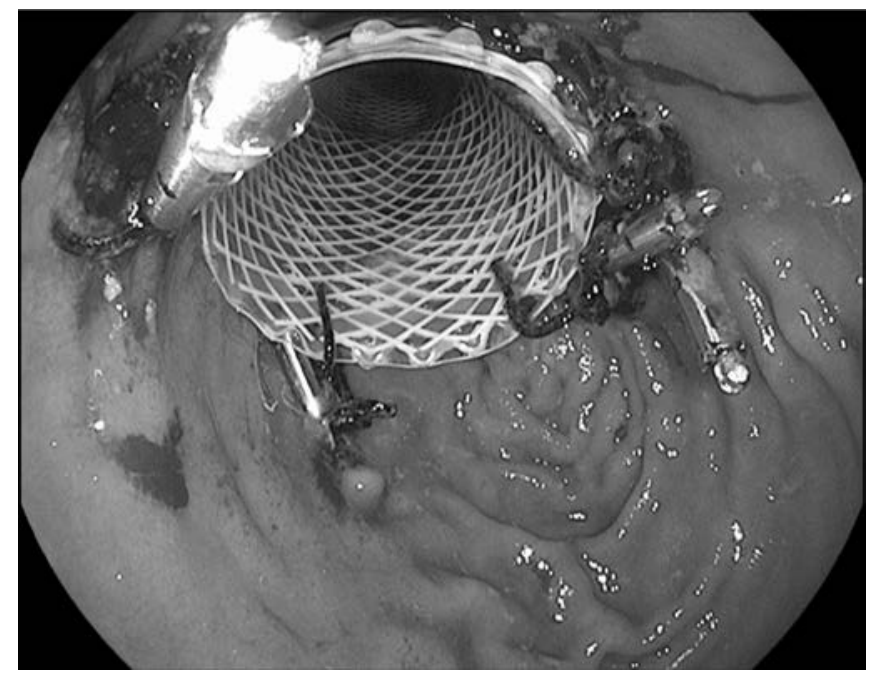

Figure 6. Single-flare stent reversal and fixation with clips and sutures.

complications (migration, restenosis, and perforation or fistulization) occurring in 67\% of patients. In the largest prospective pilot study to date, Attar et $\mathrm{al}^{12}$ noted that although stenting is logical on the basis of the principle of endoscopic stricturoplasty and is effective in relieving symptoms in the majority (60\% in their series of ileal and ileocolonic anastomotic strictures), it is plagued by spontaneous migration in $70 \%$ of cases within days of stenting, and they recommended placing the stents for only 7 days, with planned extraction after that. Thus, there does not appear to be clear evidence either way for stenting of CD fibrostenotic small bowel, anastomotic, and colonic stric-

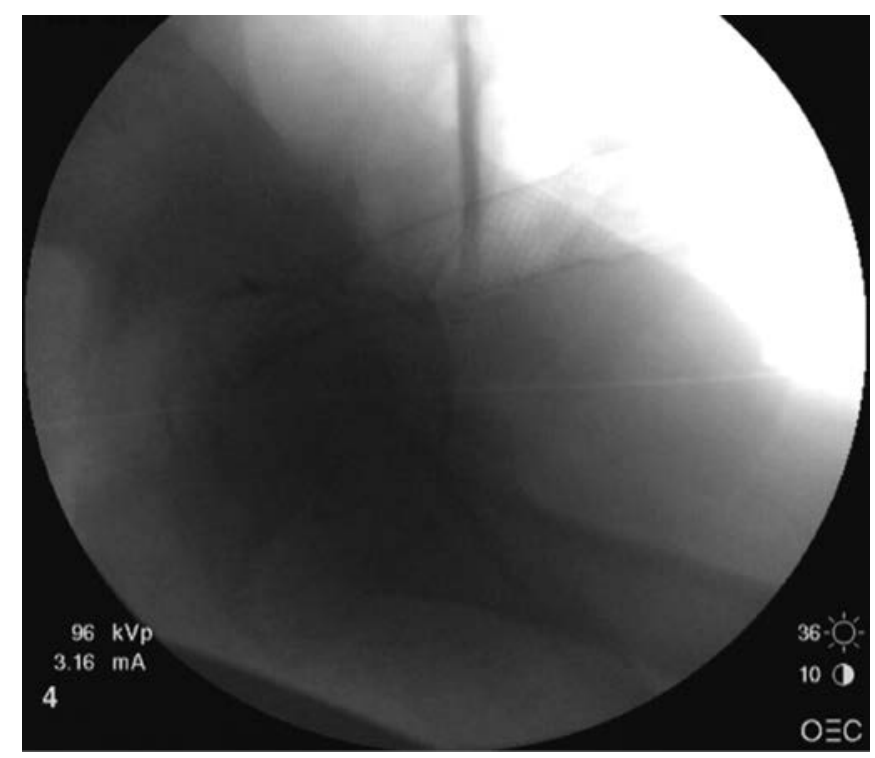

Figure 7. Fluoroscopic image of reversed single-flare stent.

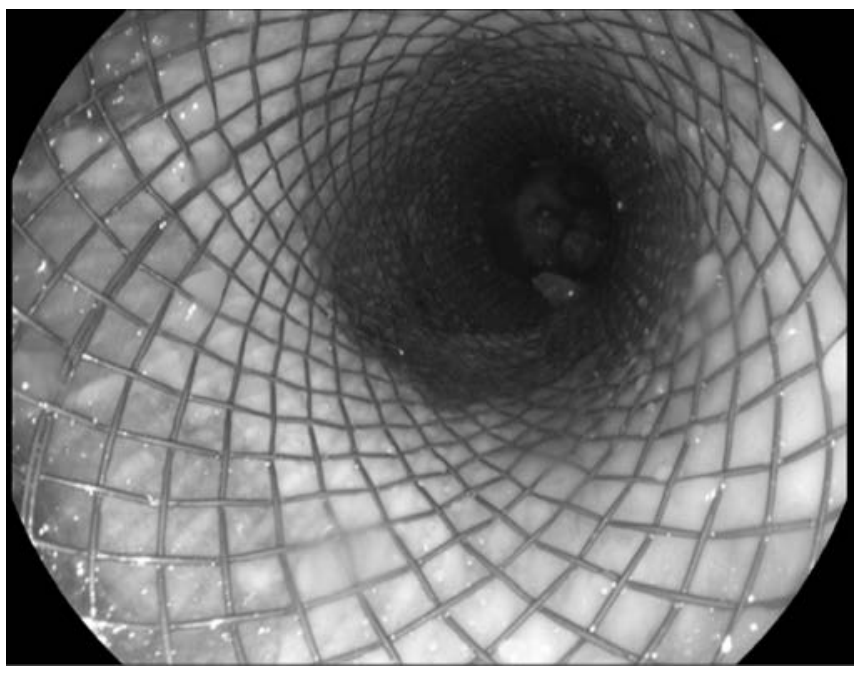

Figure 8. Partially covered nitinol double-flared stent in good position.

tures. It does represent one of the options to be considered, but care needs to be individualized to the patient, and complications are frequent; only larger prospective studies and better designed stents may provide the final answer.

In the face of recalcitrant afferent pouch limb strictures, our technique of endoscopic stenting with a partially covered stent provides a simple long-term solution. The central covered component prevents tissue ingrowth, and the uncovered ends allow tissue ingrowth and prevent migration or dislodgement. These stents can be easily placed, 
removed, adjusted, or exchanged; the patient enjoys excellent quality of life and is stoma free.

The Evolution Stent (Cook Medical) is a nitinol stent designed with uncovered dual flanges that secure the stent, potentially reducing the risk for migration or stent movement after placement, thus eliminating the need for repeat procedures. Encased inside and out with silicone, this stent is designed to prevent ingrowth and decrease food bolus impaction (it is marketed as an esophageal stent), and a "lasso" loop on the proximal end gives one the option to reposition the stent immediately after placement. The stent's retractable delivery system facilitates direct monitoring of the placement progress throughout the procedure with its "point-of-no-return" indicator, allowing a more controlled placement of the stent.

Currently, several types of self-expanding metal stents exist and vary by type of alloy, configuration, degree of shortening after release, length and diameter, type and extent of covering, delivery system, expandable force, and removability. ${ }^{13}$ Almost all are made of nitinol, an alloy of nickel and titanium, whose peculiarity is superelasticity and shape memory. The covering is polyurethane, silicone, or polytetrafluoroethylene. Almost all these stents are designed primarily for palliation of malignant dysphagia and benign esophageal strictures, stenosis, and tracheoesophageal fistula. There are no stents specifically designed for small bowel strictures, but the plethora of stents allows one to choose the type depending on the indication, the duration of use, the site, and the pathology.

\section{CONCLUSIONS}

Although our practice in this treatment modality is limited, our experience suggests that using a flexible partially covered nitinol stent with dual flares provides an effective functional outcome. Although the earlier single-flare stents needed sutures and clips distally to prevent migration, the Evolution stent needs no additional maneuvers to secure it. We believe this provides an optimal outcome and longevity of the stent. This novel treatment approach provides patients with an opportunity for management of their disease without the risk of reoperative pelvic surgery, further bowel resection, or permanent ileostomy. In our experience, the only concerns have been migration of the stent and need for replacement, either of which is more palatable than a permanent stoma. This stent and the techniques described in this article have widespread applicability not only in pouch strictures but also for nonoperative management of coloanal and low colorectal anastomosis after ultralow anterior resections for rectal cancer.

\section{References:}

1. Hueting WE, Buskens E, van der Tweel I, Gooszen HG, van Laarhoven CJ. Results and complications after ileal pouch anal anastomosis: a meta-analysis of 43 observational studies comprising 9,317 patients. Dig Surg. 2005;22:69-79.

2. Sagar PM, Dozois RR, Wolff BG. Long-term results of ileal pouch-anal anastomosis in patients with Crohn's disease. Dis Colon Rectum. 1996;39:893-898.

3. Shen B, Remzi FH, Lavery IC, Lashner BA, Fazio VW. A proposed classification of ileal pouch disorders and associated complications after restorative proctocolectomy. Clin Gastroenterol Hepatol. 2008;6(2):145-158.

4. Shen B. Diagnosis and management of postoperative ileal pouch disorders. Clin Colon Rectal Sur. 2010;23:259-268.

5. Melton GB, Fazio VW, Kiran RP, et al. Long-term outcomes with ileal pouch-anal anastomosis and Crohn's disease: pouch retention and implications of delayed diagnosis. Ann Surg. 2008; 248(4):608-616.

6. Melton GB, Kiran RP, Fazio VW, et al. Do preoperative factors predict subsequent diagnosis of Crohn's disease after ileal pouch-anal anastomosis for ulcerative or indeterminate colitis? Colorect Dis. 2010;12:1026-1032.

7. Shen B, Lian L, Kiran RP, et al. Efficacy and safety of endoscopic treatment of ileal pouch strictures. Inflamm Bowel Dis. 2011;17:2527-2535.

8. Galanduk S, Scott NA, Dozois RR, et al. Ileal pouch-anal anastomosis. Reoperation for pouch-related complications. Ann Surg. 1990;212:446-452.

9. Hassan C, Zullo A, De Francesco V, et al. Systematic review: endoscopic dilatation in Crohn's disease. Aliment Pharmacol Ther. 2007;26:1457-1464.

10. Sebastian S, Johnston S, Geoghegan T, Torreggiani W, Buckley M. Pooled analysis of the efficacy and safety of self-expanding metal stenting in malignant colorectal obstruction. Am J Gastroenterol. 2004;99:2051-2057.

11. Levine RA, Wasvary H, Kadro O. Endoprosthetic management of refractory ileocolonic anastomotic strictures after resection for Crohn's disease: report of nine-year follow-up and review of the literature. Inflamm Bowel Dis. 2012;18:506-512.

12. Attar A, Maunoury V, Vahedi K, et al; GETAID. Safety and efficacy of extractible self-expandable metal stents in the treatment of Crohn's disease intestinal strictures: a prospective pilot study. Inflamm Bowel Dis. 2012;18:1849-1854.

13. Conio M, De Ceglie A. Esophageal prostheses. In: Kozarek R, Baron T, Song H., eds. Self-Expandable Stents in the Gastrointestinal Tract. New York: Springer Science+Business Media; 2013:73-88 